\title{
Parapteronotus bonapartii (Castelnau), considerado sinônimo sênior de Parapteronotus hasemani (Ellis) (Teleostei, Apteronotidae)
}

\author{
Mauro L. Triques
}

Departamento de Zoologia, Instituto de Ciências Biológicas, Universidade Federal de Minas Gerais. Avenida Antônio Carlos 6627, Caixa Postal 486, 31270-901 Belo Horizonte, M inas Gerais, Brasil. E-mail: triques@mono.icb.ufmg.br

\begin{abstract}
Parapteronotus bonapartii (Castelnau), considered senior synonym of Parapteronotus hasemani (Ellis) (Teleostei: Apteronotidae). Parapteronotus bonapartii (Castelnau, 1855), is synonymized to Parapteronotus hasemani (Ellis, 1913), on the basis of head and body skin covered with numerous fine dark dots, a fleshy projection anterolaterally on upper lip, and adult males with elongated snout.

KEY WORDS. Freshwater electric fish, morphology, neotropical region, synonymy.
\end{abstract}

\begin{abstract}
RESUMO. Parapteronotus bonapartii (Castelnau, 1855), é sinonimizado a Parapteronotus hasemani (Ellis, 1913), com base na presença de pequenas marcas escuras sobre a pele do corpo e cabeça, uma expansão carnosa ântero-lateralmente no lábio superior e focinho alongado nos machos adultos.

PALAVRAS-CHAVE. Morfologia, peixes elétricos de água doce, região neotropical, sinonímia.
\end{abstract}

Em visita ao Muséum National d'Histoire Naturelle (Paris) em 1998, observei o holótipo de Sternarchus bonapartii Castelnau, 1855, atualmente considerado como espécie de Apteronotus (Albert 2003: 498). O holótipo encontra-se descorado, não sendo possível verificar a presença ou ausência de faixa clara dorsal ou se a região distal da nadadeira anal era escurecida (pois não havia sinal de cromatóforos); peitorais também empalidecidas, com parte distal de seus raios perdida. A nadadeira caudal foi perdida em vida e regenerada na forma de uma minúscula nadadeira. A nadadeira anal estava danificada posteriormente. As escamas da linha lateral apresentam um entalhe junto da abertura do poro e são truncadas posteriormente. Outros caracteres verificados são: boca terminal, focinho alongado cônico, perfil cefálico dorsal reto para baixo e para frente, desde uma vertical passando pelo canto da boca até uma vertical pela narina posterior; dentário com duas séries de dentes cônicos; pré-maxilar com dentes agrupados irregularmente; ânus um pouco atrás de uma vertical pela narina posterior; comprimento do focinho equivalente a $42 \%$ do comprimento cefálico. O desenho de Kaup (1856: 126, fig. 1) é fiel ao tipo, inclusive mostrando o focinho amassado anteriormente. A figura de MAGO-LECCIA (1994: 136, fig. 27) reflete a condição atual de conservação do holótipo.

Albert (2001: 77) notou que Apteronotus bonapartii (Castelnau, 1855) apresenta um focinho mais longo que aquele caracterizado por Eluıs (1913) para esta espécie, de modo que aventou a hipótese de serem espécies distintas. De acordo com LUNDBERG et al. (2000: 36), a espécie nominal Apteronotus anas é baseada em exemplares machos grandes, de focinho alongado por hipermorfose relacionada a dimorfismo sexual da espécie Apteronotus hasemani. O focinho do holótipo de Apteronotus bonapartii encontra-se em situação intermediária entre o focinho de A. hasemani e A. anas, de modo que as três espécies podem ser sinônimas. O estado de conservação do holótipo de A. bonapartii dificulta uma análise detalhada de todo o animal, mas permite um estudo conclusivo. Albert (2001: 73) erigiu um novo gênero, Parapteronotus, para incluir A. hasemani, que foi, por ele, considerada sinônimo sênior de A. anas.

O objetivo deste trabalho é demonstrar que Apteronotus bonapartii é sinônimo sênior de P. hasemani. O material de Apteronotidae utilizado neste trabalho está apresentado em TRIQUeS (2005) e informações do material tipo de P. hasemani foram obtidas da descrição original.

\section{Parapteronotus bonapartii (Castelnau, 1855) comb. nov.}

Albert (2001: 73-75; fig. 47) diagnosticou Parapteronotus (monotípico) como sendo um apteronotídeo com corpo coberto por numerosos cromatóforos escuros pequenos; margem oral do lábio superior carnoso e quadrado anteriormente; região pré-orbital que se alonga progressivamente em machos maiores que $250 \mathrm{~mm}$ de comprimento total; nadadeira caudal truncada; pedúnculo caudal curto, bem como diversos caracteres esqueléticos. TRIQUes (2005) não seguiu a sinonímia de AlbeRT (2001) e considerou P. anas separadamente de P. hasemani; de acordo com este autor, apenas P. anas apresenta coloração de fundo do corpo creme, salpicado de numerosos pontos castanho escuro, conspícuos e visíveis a olho nu (seu caráter 33), 
dentre os apteronotídeos por ele estudados. Entretanto, a descrição original de P. hasemani menciona a existência deste caráter no material tipo da espécie (ElLıs 1913: 148). O exame do holótipo de Parapteronotus bonapartii (Castelnau, 1955) permite verificar o alongamento do focinho (ilustrado em MAGOLECCIA1994: 136, fig. 27). De acordo com Kaup (1856: 127), a região ventro-lateral do corpo e o lado do filamento carnoso localizado póstero-mediano-dorsalmente no corpo apresentam pequenas marcas escuras. Segundo Kaup (1856: 126, fig. 1) ocorre uma expansão carnosa ântero-lateralmente no lábio superior, similar àquela ilustrada por AlbeRT (2001: 74, fig. 47).

As outras características de anatomia externa, diagnósticas de Parapteronotus, não podem ser verificadas no holótipo de P. bonapartii porque a sua condição atual de conservação não permite a visualização ou porque o animal foi mutilado e regenerado em vida. Entretanto, as características apresentadas por KaUP (1856) e o alongamento do focinho são suficientes para sinonimizar P. hasemani a P. bonapartii com clareza, uma vez que esta combinação não ocorre em nenhuma outra espécie de apteronotídeo e não são conhecidas diferenças entre elas.

Parapteronotus macrostomus é a outra espécie do gênero (TRIQUEs 2005) e difere de P. bonaparti por apresentar escamas da linha lateral circulares, levemente alongadas horizontalmente, com margem posterior afilada dorsalmente e oblíqua ventralmente, sem ental he junto da abertura do poro e colorido corporal castanho escuro uniforme. A pteronotus apurensis, A. macrolepis e Apteronotus rostratus têm focinho moderadamente alongado e poderiam ser confundidas com P. bonapartii, mas diferem desta porque possuem o perfil anterior do focinho arredondado. Apteronotus mariae apresenta perfil dorsal do focinho curvo para baixo (MAGO-LECCIA 1994: 139, fig. 32). A pteronotus leptorhynchus tem o focinho similar ao de P. bonapartii, mas dorsalmente apresenta suave convexidade (M AGo-LECCIA 1994: 137, fig. 30, TRIQUES 2005: 130, fig. 13) e seu colorido corporal é castanho escuro. Lábio quadrado anteriormente ocorre também em Orthosternarchus tamandua e Sternarchella orthos (TRIQUes 2005), mas uma série de outros caracteres os coloca em outros gêneros (e.g. ALBERT 2001, TRIQUEs 2005, dentre muitos outros). Este caráter não mais está presente de forma conspícua no holótipo, pois este foi preservado em álcool, mas foi representado como projeção ânterolateral arredondada do lábio superior em KAUP (1856) e é visivelmente arredondada em alguns exemplares desta espécie (ALBERT 2001: 74-75, fig. 47 b, c). Algumas espécies de apteronotídeos de focinho alongado e aproximadamente reto não foram estudadas por TRIQUes (2005) e são diferenciadas de P. bonapartii subseqüentemente. Apteronotus caudimaculosus Santana, 2003, apresenta colorido escuro tendendo ao negro, com faixas despigmentadas transversais na parte posterior do corpo, enquanto que P. bonapartii não apresenta colorido escuro. Apteronotus eschmeyeri Santana et al., 2004, apresenta colorido manchado, com faixas despigmentadas transversais na parte posterior do corpo, enquanto que P. bonapartii não apresenta colorido manchado nem faixa transversal clara no corpo.
O formato e o grau de alongamento do focinho do holótipo de P. bonapartii indica claramente tratar-se de um indivíduo em um estágio intermediário entre indivíduos machos menores e fêmeas (descritos como A. hasemani) e indivíduos machos adultos maiores (descritos como A. anas). O colorido do holótipo foi descrito por Kaup (1856) e também é compatível com esta hipótese, uma vez que na descrição original de Parapteronotus hasemani (ElLıs 1913), o autor diz que o colorido de fundo é creme com diminutas marcas escuras sobre o corpo e regiões lateral e ventral da cabeça, o que pode ser visualizado em AlBERT (2001: 75, fig. 47c). A projeção carnosa ântero-lateral do lábio, ilustrada também por KAuP (1856) é outro reforço para a sinonímia, uma vez que foi ilustrada para P. hasemani por ElLIS (1913: pl. 23, fig. 1).

Assim, fica evidenciado que o holótipo de P. bonapartii somente pode ser correlacionado como uma forma intermediária do desenvolvimento ontogenético de $\mathrm{P}$. hasemani, dentre as espécies de Apteronotidae, sendo seu sinônimo sênior.

\section{AGRADECIMENTOS}

Aos colegas que me disponibilizaram exemplares sob seus cuidados: José Lima de Figueiredo, Osvaldo Takeshi Oyakawa, J.-C. Hureau, Richard P. Vari, Stanley H. Weitzman, William Fink, Francisco R. Provenzano, John G. Lundberg e Susan S. Jewett. Ao revisor anônimo, pela leitura crítica e sugestões que aperfeiçoaram o manuscrito. A Frederico Miranda de Queiroz, pela elaboração do arquivo em pdf do manuscrito. À FAPEMIG (CRA 2146/97), pelos recursos proporcionados.

\section{REFERÊNCIAS BIBLIOGRÁFICAS}

AlberT, J.S. 2001. Species diversity and phylogenetic systematics of American Knifefishes (Gymnotiformes, Teleostei). Miscellaneous Publications Museum of Zoology, University of Michigan (190): $\mathrm{VI}+127$.

Albert, J. S. 2003. Family Apteronotidae, p. 497-502. In: R.E. ReIS; S.O. Kullander \& C. J. FerRarIS JR (Eds). Check List of the freshwater fishes of South and Central America. Porto Alegre, EDIPUCRS, XII+729p.

Castelnau, F.L. DE L. DE. 1855. Animaux nouveaux ou rares recueillis pendant l'expedition dans les parties centrales de l'Amérique du Sud, de Rio de Janeiro a Lima, et de Lima au Para; exëcutée par ordre du gouvernement français pendant les années 1843 a 1847. Paris, Chez $P$. Bertrand, vol. 7, pt. 2, 112p.

ELLIS, M.M. 1913. The gymnotid eels of tropical America. Memoirs of Carnegie Museum 6: 109-211.

KaUP, J.J. 1856. Family Gymnotidae, p. 124-142. In: J.J. KaUP (Ed.). Catalogue of Apodal fish in the collection of the British Museum. London, British Museum, 163p.

Lundberg, J.G.; M. Kottelat; G.R. Smith; M.L.J. Stiassny \& A.C. GILL. 2000. So many fishes, so little time: an overview of recent ichthyological discovery in continental waters.

Revista Brasileira de Zoologia 24 (1): 84-86, março 2007 
Annals of the Missouri Botanical Garden 87: 26-62.

MAGO-LECCIA, F. 1994. Electric fishes of the continental waters of America. Caracas, Fundacion par el Desarrollo delas Ciencias Fisicas, Matematicas y Naturales, 223p.

SANTANA, C.D. 2003. Apteronotus caudimaculosus sp. nov. (Gymnotiformes: Apteronotidae), a sexually dimorphic black gost knifefish from the Pantanal, Western Brazil, with a note on the monophyly of A. albifrons complex. Zootaxa (252):
1-11.

Santana, C.D.; J.A. Maldonado-Ocampo; W. Severi \& G.N. Mendes. 2004. A pteronotus eschmeyeri, a new species of ghost knifesfish from the Magdalena Basin, Colômbia (Gymnotiformes: Apteronotidae). Zootaxa (410): 1-11.

TRIQUes, M.L. 2005. Análise cladística de caracteres de anatomia externa e esquelética de Apteronotidae (Teleostei: Gymnotiformes). Lundiana 6 (2): 121-149.

Recebido em 03.V.2006; aceito em 27.II.2007. 